TRANSACTIONS OF THE

AMERICAN MATHEMATICAL SOCIETY

Volume 278, Number 2, August 1983

\title{
UNIFORMLY EXHAUSTIVE SUBMEASURES AND NEARLY ADDITIVE SET FUNCTIONS
}

BY

\author{
N. J. KALTON ${ }^{1}$ AND JAMES W. ROBERTS ${ }^{2}$
}

\begin{abstract}
Every uniformly exhaustive submeasure is equivalent to a measure. From this, we deduce that every vector measure with compact range in an $F$-space has a control measure. We also show that $c_{0}$ (or any $\varrho_{\infty}$-space) is a $\mathcal{K}$-space, i.e. cannot be realized as the quotient of a nonlocally convex $F$-space by a one-dimensional subspace.
\end{abstract}

1. Introduction. The purpose of this paper is to study and provide partial solutions for two questions which arise naturally in the study of $F$-spaces (complete metrizable topological vector spaces). Both of these questions have attractive formulations independent of $F$-space theory.

It is well known that the control measure problem for countably additive vector measures is equivalent to a classical problem of Maharam [11] on the existence of a control measure for a continuous submeasure. We now describe our results on this problem, giving first a different but equivalent formulation of the Maharam problem in terms of exhaustive submeasures.

Let $\mathbb{Q}$ be an algebra of sets. Throughout this paper a measure $\lambda$ on $\mathbb{Q}$ is, unless otherwise stated, positive and finitely additive. A submeasure $\phi: \mathbb{Q} \rightarrow \mathbf{R}$ is a map satisfying

(i) $\phi(\varnothing)=0$,

(ii) $\phi(A) \leqslant \phi(B), A \subset B$

(iii) $\phi(A \cup B) \leqslant \phi(A)+\phi(B), A, B \in \mathbb{Q}$.

$\phi$ is exhaustive if

(iv) $\lim _{n \rightarrow \infty} \phi\left(A_{n}\right)=0$

for every disjoint sequence $\left(A_{n}: n \in \mathbf{N}\right)$ in $Q$.

Two submeasures $\phi$ and $\psi$ are equivalent if $\phi\left(A_{n}\right) \rightarrow 0$ if and only if $\psi\left(A_{n}\right) \rightarrow 0$. Maharam's problem asks if every exhaustive submeasure is equivalent to a measure.

A submeasure $\phi$ is pathological if whenever $\lambda$ is a measure with $0 \leqslant \lambda \leqslant \phi$ then $\lambda=0$. If there is an exhaustive submeasure not equivalent to a measure then there is a nontrivial exhaustive pathological submeasure. Examples of pathological submeasures have been constructed in $[\mathbf{2}, 14$ and 21$]$; unfortunately these are not exhaustive.

Received by the editors July 20, 1982 and, in revised form, August 26, 1982.

1980 Mathematics Subject Classification. Primary 28A60, 28A45, 46A15.

Key words and phrases. Submeasures, control measure, twisted sum.

'Research supported by the NSF-grant MCS-8001852.

${ }^{2}$ Research supported by the NSF-grant MCS-7901044. 
In [21] Talagrand defines a submeasure $\phi$ to be uniformly exhaustive if given $\varepsilon>0$ there exists $n \in \mathbf{N}$ such that for any disjoint sets $A_{1}, A_{2}, \ldots, A_{n}$,

$$
\min _{1 \leqslant i \leqslant n} \phi\left(A_{i}\right) \leqslant \varepsilon .
$$

Clearly if $\phi$ is equivalent to a measure it is also uniformly exhaustive. Our main result, which answers a question of Talagrand, is that $\phi$ is equivalent to a measure if and only if it is uniformly exhaustive. This implies, for example, that every vector measure with relatively compact range has a control measure.

The second question was raised in [7]. In [7] it was asked whether there is a nonlocally convex $F$-space $X$ containing an uncomplemented line $L$ such that $X / L \cong c_{0}$. This can be reduced to a problem on set functions [8]. We answer this by showing that there is a universal constant $C<45$ so that whenever $Q$ is an algebra of sets and $f: \mathbb{Q} \rightarrow \mathbf{R}$ is a map satisfying $|f(A \cup B)-f(A)-f(B)| \leqslant 1$ whenever $A \cap B=\varnothing$, then there is an additive set-function $\mu: \mathbb{Q} \rightarrow \mathbf{R}$ with $|f(A)-\mu(A)| \leqslant C$, $A \in \mathbb{Q}$. This implies that if $X / L \cong c_{0}$ then $X$ is locally convex and so $L$ is complemented (and $X \cong c_{0}$ ). Similar conclusions hold for any $\mathcal{L}_{\infty}$-space.

The main technique employed is the idea of a concentrator. We describe this in $\S 2$. As certain types of graphs these have been studied in several recent papers (see [4] and associated references). In fact we need only very elementary facts about concentrators, and our requirements are satisfied by Proposition 2.1, which was shown to us by E. Szemeredi. Although this is sufficient for our qualitative results, the constant in Theorem 4.1 can be improved by using a more complicated result of Pippenger [13, Proposition 2.2].

Finally we recall for future reference that an F-norm on a real vector space is a map $x \mapsto\|x\|$ satisfying

(i) $\|x\|>0, x \neq 0$,

(ii) $\lim _{t \rightarrow 0}\|t x\|=0, x \in X$,

(iii) $\|a x\| \leqslant\|x\|,|a| \leqslant 1, x \in X$,

(iv) $\|x+y\| \leqslant\|x\|+\|y\|, x, y \in X$.

A quasinorm $x \mapsto\|x\|$ satisfies

(i) $\|x\|>0, x \neq 0$,

(ii) $\|a x\|=|a|\|x\|, a \in \mathbf{R}, x \in X$,

(iii) $^{\prime}\|x+y\| \leqslant C(\|x\|+\|y\|), x, y \in X$

for some constant $C$.

A quasinorm is $p$-subadditive $(0<p \leqslant 1)$ if $\|x\|^{p}$ is an $F$-norm. A locally bounded $F$-space can be topological by a $p$-subadditive quasinorm for some $p$, and is called a quasi-Banach space.

2. Concentrators. If $m \in \mathbf{N}$ then $[m]$ will denote the set $\{1,2, \ldots, m\}$ and $2^{[m]}$ is the collection of all subsets of $[m]$. If $m, p \in \mathbf{N}$ and $R:[m] \rightarrow 2^{[p]}$ is a map, then for $E \in 2^{[m]}$ we define

$$
R[E]=\bigcup_{j \in E} R(j) .
$$

For any set $A$ let $|A|$ denote the number of elements in $A$. 
If $m, p, q, r \in \mathbf{N}$ where $m \geqslant p \geqslant q$, then we say that a map $R:[m] \rightarrow 2^{[p]}$ is an ( $m, p, q, r)$-concentrator if

(i) $\sum_{j=1}^{m}|R(j)| \leqslant r m$,

(ii) $|R[E]| \geqslant|E|$ whenever $E \in 2^{[m]}$ with $|E| \leqslant q$.

$R$ may be alternatively considered as a bipartite graph or a subset of the product space $[m] \times[p]$. In the case $p=q$ concentrators have been studied in a number of articles (see $[4,12,13])$. The following result is apparently well known and its proof was shown to the authors by E. Szemerédi.

Proposition 2.1. Suppose $m, p, q, r \in \mathbf{N}$ with $3 \leqslant r \leqslant q \leqslant p \leqslant m$, and $2 e^{2} m q^{r-2}$ $\leqslant p^{r-1}$. Then there is an $(m, p, q, r)$-concentrator.

Proof. Let $\Omega$ be the set of all maps $R:[m] \rightarrow 2^{[p]}$ so that $|R(j)|=r$ for $j \in[m]$. Then

$$
|\Omega|=\left(\begin{array}{c}
p \\
r
\end{array}\right)^{m}
$$

Let $P$ be the probability measure on $\Omega$ given by

$$
P(\Lambda)=|\Lambda| /|\Omega| \text {. }
$$

Suppose $F \in|p|$ with $|F|=n$ where $r \leqslant n \leqslant q$. Then

$$
P(R(j) \subset F)=\left(\begin{array}{l}
n \\
r
\end{array}\right) \cdot\left(\begin{array}{c}
p \\
r
\end{array}\right)^{-1} \leqslant \frac{n^{r}}{p^{r}} .
$$

If $E \subset[m]$ with $|E|=n$ then

$$
P(R[E] \subset F)=\prod_{j \in E} P(R(j) \subset F) \leqslant \frac{n^{r n}}{p^{r n}} .
$$

Now let $\Lambda$ be the set of $R$ so that $|R(E)| \leqslant|E|$ for some $E \in 2^{[r]}$ with $|E| \leqslant q$. Then

$$
P(\Lambda) \leqslant \sum_{\substack{r \leqslant|E| \leqslant q \\
|F|=|E|}} P(R[E] \subset F) \leqslant \sum_{n=r}^{q}\left(\begin{array}{c}
m \\
n
\end{array}\right)\left(\begin{array}{c}
p \\
n
\end{array}\right) \frac{n^{r n}}{p^{r n}}
$$

We next use the estimate $n ! \geqslant n^{n} e^{-n}$ to obtain

$$
P(\Lambda) \leqslant \sum_{n=r}^{q} \frac{m^{n} e^{2 n} n^{(r-2) n}}{p^{(r-1) n}} \leqslant \sum_{n=r}^{q}\left(\frac{1}{2}\right)^{n}<1 .
$$

Now pick any $R \notin \Lambda$ and $R$ is an $(m, p, q, r)$-concentrator.

Proposition 2.1 will supply all we need in this paper for qualitative results. However for certain quantitive results we observe that a more delicate argument of a similar nature has been used by Pippenger [13] to show:

Proposition 2.2. For every $m \in \mathbf{N}$ there is a $(6 m, 4 m, 3 m, 6)$-concentrator.

Fix $r \in \mathbf{N}$ and $0<\delta, \varepsilon<1$. We shall say that $H(r, \delta, \varepsilon)$ holds if there are sequences of integers $\left\{m_{k}\right\},\left\{p_{k}\right\},\left\{q_{k}\right\}$ so that $m_{k} \rightarrow \infty, p_{k} / m_{k} \leqslant \delta$ and $q_{k} / m_{k} \geqslant \varepsilon$, 
and for each $k$ there is an $\left(m_{k}, p_{k}, q_{k}, r\right)$-concentrator. For fixed $r \in \mathbf{N}$ and $\varepsilon>0$ let

$$
\theta(r, \varepsilon)=\inf \{\delta: H(r, \delta, \varepsilon) \text { holds }\} .
$$

Proposition 2.3. (i) $\theta(r, \varepsilon) \leqslant\left(2 e^{2}\right)^{1 /(r-1)} \varepsilon^{(r-2) /(r-1)}(r \geqslant 3)$.

(ii) $\theta\left(6, \frac{1}{2}\right) \leqslant \frac{2}{3}$.

These are immediate from the preceding propositions.

3. Applications to submeasures. Let $\Omega$ be an abstract set and let $\mathbb{Q}$ be any algebra of subsets of $\Omega$. For $A \in \mathbb{Q}$ we denote by $1_{A}$ the characteristic function of $A$, so that $1_{A}: \Omega \rightarrow \mathbf{R}$.

LEMma 3.1. Let $\phi: \mathbf{A} \rightarrow \mathbf{R}$ be a submeasure with the property that for some $\alpha, \beta \geqslant 0$ whenever $n \in \mathbf{N}$, and $A_{1}, A_{2}, \ldots, A_{n} \in \mathbb{Q}$ are disjoint then $\sum_{i=1}^{n} \phi\left(A_{i}\right) \leqslant \alpha n+\beta$. Suppose $B_{1}, \ldots, B_{m} \in \mathbb{Q}$ and $m^{-1} \sum_{i=1}^{m} 1_{B_{i}} \geqslant(1-\varepsilon) 1_{\Omega}$. Then for $r \in \mathbf{N}$ with $r \geqslant 3$,

$$
\frac{1}{m} \sum_{i=1}^{m} \phi\left(B_{i}\right) \geqslant \phi(\Omega)-\alpha r-\beta \theta(r, \varepsilon) .
$$

Proof. Suppose first $B_{1}, \ldots, B_{m} \in \mathbb{Q}$ with

$$
\frac{1}{m} \sum_{i=1}^{m} 1_{B_{i}} \geqslant(1-\varepsilon) 1_{\Omega}
$$

and there is an $(m, p, q, r)$-concentrator $R:[m] \rightarrow 2^{[p]}$ where $q / m \geqslant \varepsilon$.

Let $\mathcal{E}$ be the collection of $E \in 2^{[m]}$ with $|E| \leqslant q$. For every $E \rightarrow \mathcal{E}$ there is one-one map $f_{E}: E \rightarrow[p]$ with $f_{E}(j) \in R(j)$ for $j \in E$; this follows from Hall's Marriage Lemma [5].

For $E \in \mathcal{E}$ define

$$
C_{E}=\bigcap_{k \in E}\left(\Omega \backslash B_{k}\right) \cap \bigcap_{k \notin E} B_{k} .
$$

By hypothesis on $B_{1}, \ldots, B_{m}, \cup_{E \in \mathcal{E}} C_{E}=\Omega$.

For $i \in[m]$ and $j \in[p]$ let

$$
A_{i j}=\bigcup\left(C_{E}: i \in E, f_{E}(i)=j\right) .
$$

$A_{i j} \neq \varnothing$ implies that $j \in R(i)$ and hence the number of nonempty sets $\left\{A_{i j}\right\}$ is at most $r m$.

For fixed $j \in[p]$, the sets $\left\{A_{i j} ; i \in[m]\right\}$ are disjoint. Indeed if $A_{i j}$ and $A_{k j}$ intersect then for some $E \in \mathcal{E}, C_{E} \subset A_{i j}$ and $C_{E} \subset A_{k j}$ so that $i, k \in E$ and $f_{E}(i)=f_{E}(k)=j$. Thus $i=k$. If $n_{j}$ denotes the number of nonempty sets $\left\{A_{i j} ; i \in\right.$ $[m]\}$, we have

$$
\sum_{i=1}^{m} \phi\left(A_{i j}\right) \leqslant \alpha n_{j}+\beta
$$

and hence

$$
\sum_{j=1}^{p} \sum_{i=1}^{m} \phi\left(A_{i j}\right) \leqslant \alpha \sum_{j=1}^{p} n_{j}+\beta p \leqslant \alpha m r+\beta p .
$$


On the other hand for $i \in[m]$,

$$
\sum_{j=1}^{p} \phi\left(A_{i j}\right) \geqslant \phi\left(\bigcup_{j \in[p]} A_{i j}\right)=\phi\left(\bigcup_{i \in E} C_{E}\right)=\phi\left(\Omega \backslash B_{i}\right) \geqslant \phi(\Omega)-\phi\left(B_{i}\right) .
$$

Thus

$$
m \phi(\Omega)-\sum_{i=1}^{m} \phi\left(B_{i}\right) \leqslant \alpha m r+\beta p
$$

so that

$$
\frac{1}{m} \sum_{i=1}^{m} \phi\left(B_{i}\right) \geqslant(\phi(\Omega)-\alpha r)-\beta \frac{p}{m} .
$$

Now suppose instead that there is an $(n, p, q, r)$-concentrator where $m \leqslant n$ and $q \geqslant \varepsilon n$. We can apply the above argument to the collection of sets $\left\{B_{1}, \ldots, B_{m}\right\}$ repeated $s=[n / m]$ times and $n-m s$ copies of $\Omega$. We obtain

$$
\frac{m s}{n} \cdot \frac{1}{m} \sum_{i=1}^{m} \phi\left(B_{i}\right)+\frac{n-m s}{n} \phi(\Omega) \geqslant \phi(\Omega)-\alpha r-\beta \frac{p}{n} .
$$

Thus if $H(r, \delta, \varepsilon)$ holds we obtain

$$
\frac{1}{m} \sum_{i=1}^{m} \phi\left(B_{i}\right) \geqslant \phi(\Omega)-\alpha r-\beta \delta
$$

and so

$$
\frac{1}{m} \sum_{i=1}^{m} \phi\left(B_{i}\right) \geqslant \phi(\Omega)-\alpha r-\beta \theta(r, \varepsilon) .
$$

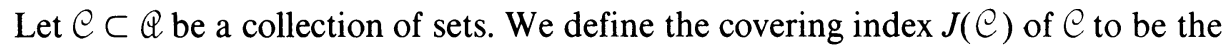
supremum of all $\tau \geqslant 0$ so that there exists $C_{1}, \ldots, C_{n} \in \mathcal{C}$ with

$$
\tau 1_{\Omega} \leqslant \frac{1}{n} \sum_{i=1}^{n} 1_{C_{i}} .
$$

If $\phi$ is a submeasure let

$$
\mathcal{C}(\phi ; \delta)=\{C: \phi(C) \leqslant \delta\}
$$

The following proposition collects the facts on the covering index that we shall require:

Proposition 3.2. (i) (Kelley [10]). If $\subset \subset \mathbb{Q}$ is any subset there is a measure $\mu$ : $Q \rightarrow \mathbf{R}$ with $\mu(C) \leqslant J(\mathcal{C})(C \in \mathcal{C})$ and $\mu(\Omega)=1$.

(ii) (Christensen [1]). If $\phi$ is a nontrivial pathological submeasure, $J[C(\phi ; \delta)]=1$ for every $\delta>0$.

THEOREM 3.3. Let $\phi$ be a uniformly exhaustive pathological submeasure. Then $\phi=0$.

Proof. Suppose $\phi(\Omega)>0$. Then there exists $N \in \mathbf{N}$ so that if $A_{1}, \ldots, A_{N+1}$ are disjoint

$$
\min _{1 \leqslant i \leqslant N+1} \phi\left(A_{i}\right)<\frac{1}{5} \phi(\Omega) .
$$


Thus for any disjoint collection $\left\{A_{1}, \ldots, A_{n}\right\}$ we have

$$
\sum_{i=1}^{n} \phi\left(A_{i}\right) \leqslant \frac{1}{5} n \phi(\Omega)+N \phi(\Omega)
$$

Let $\delta=\frac{1}{5} \phi(\Omega)$ and suppose $B_{1}, \ldots, B_{m} \in \mathcal{C}(\phi ; \delta)$ and

$$
\frac{1}{m} \sum_{i=1}^{m} 1_{B_{i}} \geqslant(1-\eta) 1_{\Omega} \text {. }
$$

Then by Lemma 3.1 with $r=3$,

$$
\theta(3, \eta) N \phi(\Omega) \geqslant \frac{1}{5} \phi(\Omega) .
$$

By Proposition 2.3, there exists $\varepsilon>0$ so that $N \theta(3, \varepsilon)<\frac{1}{5}$. Thus

$$
J(\bigodot(\phi ; \delta)) \leqslant 1-\frac{1}{2} \varepsilon .
$$

By Proposition 3.2, this is a contradiction, so that we must have $\phi=0$.

TheOREM 3.4. Let $\phi: \mathbb{Q} \rightarrow \mathbf{R}$ be a submeasure. Then a necessary and sufficient condition that $\phi$ is equivalent to a measure is that $\phi$ be uniformly exhaustive.

Proof. Of course if $\phi$ is equivalent to a measure, $\phi$ must be uniformly exhaustive. The converse is a standard deduction from Theorem 3.3 (cf. [1 and 20]) which we give for the sake of completeness. The argument is based on one used by Talagrand [21].

Let $\varepsilon_{n} \rightarrow 0$ be such that for every disjoint collection $A_{1}, \ldots, A_{n} \in \mathbb{Q}$ we have $\min _{1 \leqslant i \leqslant n} \phi\left(A_{i}\right) \leqslant \varepsilon_{n}$. Choose $\left(\mu_{i}: i \in I\right)$ a maximal collection of nontrivial measures so that $0 \leqslant \mu_{i} \leqslant \phi$, and if $i \neq j$,

$$
\mu_{i} \wedge \mu_{j}(\Omega)=\inf _{A \in \mathbb{Q}}\left(\mu_{i}(A)+\mu_{j}(\Omega \backslash A)\right)=0 .
$$

For any $\mu_{i_{1}}, \ldots, \mu_{i_{n}}$ and $\eta>0$ we can find $A_{1}, \ldots, A_{n} \in \mathbb{Q}$ disjoint so that $\mu_{i_{j}}\left(A_{j}\right) \geqslant$ $\mu_{i_{j}}(\Omega)-\eta, j=1,2, \ldots, n$. Hence

$$
\min _{j \leqslant n} \mu_{i_{j}}(\Omega) \leqslant \varepsilon_{n}+\eta
$$

Since $\eta>0$ is arbitrary,

$$
\min _{j \leqslant n} \mu_{i_{j}}(\Omega) \leqslant \varepsilon_{n}
$$

It follows that the collection $\left(\mu_{i}: i \in I\right)$ is either finite or countably infinite. Hence there are $\left(c_{i}: i \in I\right)$ so that $c_{i}>0$ and $\Sigma c_{i}=1$. Let $\mu=\sum_{i \in I} c_{i} \mu_{i}$. Then $\mu$ is a measure on $Q$ which is equivalent to $\phi$.

In fact $\phi\left(A_{n}\right) \rightarrow 0$ implies $\mu_{i}\left(A_{n}\right) \rightarrow 0$ for every $i \in I$ and hence $\mu\left(A_{n}\right) \rightarrow 0$. Conversely suppose $\mu\left(A_{n}\right) \leqslant 2^{-n}$ and define

$$
\psi(B)=\lim _{m} \lim _{n} \phi\left(B \cap \bigcup_{k=m+1}^{n} A_{k}\right) .
$$

$\psi$ is a uniformly exhaustive submeasure on $\mathbb{Q}$. Suppose $\nu$ is a measure on $\mathbb{Q}$ so that $0 \leqslant \nu \leqslant \psi$. 
Since $\phi$ is exhaustive, for any $m \in \mathbf{N}$,

$$
\lim _{n \rightarrow \infty} \lim _{p \rightarrow \infty} \phi\left(\bigcup_{n+1}^{p} A_{k} \backslash \bigcup_{m+1}^{n} A_{k}\right)=0 .
$$

Thus

$$
\lim _{n \rightarrow \infty} \psi\left(\Omega \backslash \bigcup_{m+1}^{n} A_{k}\right)=0
$$

and hence

$$
\lim _{n \rightarrow \infty} \nu\left(\Omega \backslash \bigcup_{m+1}^{n} A_{k}\right)=0
$$

Now, for $i \in I$,

$$
\nu \wedge \mu_{i}(\Omega) \leqslant \nu\left(\Omega \backslash \bigcup_{m+1}^{n} A_{k}\right)+c_{i}^{-1} \cdot 2^{-m}
$$

so that $\nu \wedge \mu_{i}=0$ for $i \in I$. By the maximality of $I$, we conclude $\nu=0$ and hence $\psi$ is pathological. Thus $\psi=0$ so that $\lim _{m \rightarrow \infty} \phi\left(A_{m}\right)=0$.

4. Approximately additive set functions. Let $\mathcal{Q}$ be an algebra of subsets of a set $\Omega$ and let $f: \mathbb{Q} \rightarrow \mathbf{R}$ be any function. We shall say that $f$ is $\Delta$-approximately additive if $f(\varnothing)=0$ and given $A, B \in \mathbb{Q}$ disjoint we have $|f(A \cup B)-f(A)-f(B)| \leqslant \Delta$. The following theorem solves a problem posed by the first author (explicitly in [8] and implicitly in [7]).

THEOREM 4.1. There is a universal constant $K<45$ with the property that if $f$ : $\mathbb{Q} \rightarrow \mathbf{R}$ is $\Delta$-approximately additive there is an additive function $\mu: \mathbb{Q} \rightarrow \mathbf{R}$ with $|f(A)-\mu(A)| \leqslant K \Delta$.

Proof. It obviously suffices to consider the case $\Delta=1$. We also note that it suffices to consider the case of a finite algebra $Q$. Indeed suppose the theorem is proved for every finite algebra and that $Q$ is an infinite algebra. Then for every finite subalgebra $\mathscr{F}$ of $\mathscr{Q}$ there is an additive map $\mu_{\mathscr{F}}: \mathscr{F} \rightarrow \mathbf{R}$ with $\left|f(A)-\mu_{\mathscr{F}}(A)\right| \leqslant K$, $A \in \mathscr{F}$. Extend each $\mu_{\mathscr{F}}$ to $\mathscr{Q}$ by setting $\mu_{\mathscr{F}}(A)=0$ for $A \in \mathbb{Q} \backslash \mathscr{F}$. Order the subalgebras $\mathscr{F}$ by inclusion. Then the set $\left\{\mu_{\mathscr{F}}\right\}$ is contained in the compact subset $\{g:|g(A)| \leqslant|f(A)|+K\}$ of $\mathbf{R}^{\mathfrak{Q}}$ and so has a convergent subnet. The limit $\mu$ of this subnet is the required additive map $\mu: \mathbb{Q} \rightarrow \mathbf{R}$ with $|f(A)-\mu(A)| \leqslant K, A \in \mathbb{Q}$.

Thus we suppose $Q$ is finite and, in fact, $Q=2^{\Omega}$. For any function $g: \mathbb{Q} \rightarrow \mathbf{R}$ with $g(\varnothing)=0$ we let

$$
V(g)=\max _{A, B \subset \Omega}(g(A)-g(B)) .
$$

If $f: 2^{\Omega} \rightarrow \mathbf{R}$ is 1-approximately additive, select an additive $\mu: 2^{\Omega} \rightarrow \mathbf{R}$ so that $V(f-\mu)$ is minimized. Let $g=f-\mu$, and suppose

$$
\max _{A \in \mathbb{Q}} g(A)=a, \quad \min _{A \in \mathbb{Q}} g(A)=-b
$$


where $a \geqslant b$ (if $b>a$ consider instead $-g$ in the ensuing argument). Then

$$
|f(A)-\mu(A)| \leqslant a, \quad A \in \mathbb{Q} .
$$

Note that $g$ is 1-approximately additive.

Select $S \subset \Omega$ so that $g(S)=a$. If $B \subset S$ then $g(B) \geqslant g(S)-g(S \backslash B)-1 \geqslant-1$. Now define $\phi: 2^{S} \rightarrow \mathbf{R}$ by

$$
\begin{aligned}
\phi(A) & =1+\sup _{B \subset A} g(B), \quad A \neq \varnothing, \\
& =0, \quad A=\varnothing .
\end{aligned}
$$

Then $\phi$ is a submeasure. If $A_{1}, \ldots, A_{n} \subset S$ are disjoint then there exist $B_{i} \subset A_{i}$ $(1 \leqslant i \leqslant n)$ so that

$$
\begin{aligned}
\sum_{i=1}^{n} \phi\left(A_{i}\right) & =n+\sum_{i=1}^{n} g\left(B_{i}\right) \\
& \leqslant n+\sum_{i=1}^{n} g\left(B_{1} \cup \cdots \cup B_{n}\right)+n-1 \leqslant 2 n+(a-1) .
\end{aligned}
$$

Note also that $\phi(S)=a+1$.

We now estimate $J(\mathcal{C})$ where $\mathcal{C}=\mathcal{C}\left(\phi ; 4 \frac{1}{2}\right)$. Suppose $\lambda: 2^{S} \rightarrow \mathbf{R}$ is a measure satisfying $\lambda(S)=1$ and $\lambda(A)<\frac{1}{2}$ whenever $A \in \mathcal{C}$. We consider the map $h: 2^{\Omega} \rightarrow \mathbf{R}$ given by

$$
h(A)=g(A)-\lambda(A \cap S), \quad A \subset \Omega .
$$

Then by the original choice of $\mu$ we have $V(h) \geqslant a+b$. However we shall show that

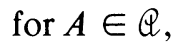

$$
-b-\frac{1}{2}<h(A)<a-\frac{1}{2},
$$

and thus obtain a contradiction.

Suppose $h(A) \geqslant a-\frac{1}{2}$, then $g(A) \geqslant a-\frac{1}{2}$. Now

$$
g(A \backslash S) \leqslant 1+g(A \cup S)-g(S) \leqslant 1
$$

so that

$$
g(A \cap S) \geqslant g(A)-g(A \backslash S)-1 \geqslant a-\frac{5}{2} .
$$

If $B \subset S \backslash A$,

$$
g(B) \leqslant g((A \cap S) \cup B)-g(A \cap S)+1 \leqslant \frac{7}{2}
$$

so that $\phi(S \backslash A) \leqslant \frac{9}{2}$. Hence $\lambda(S \backslash A)<\frac{1}{2}$ and $\lambda(A \cap S)>\frac{1}{2}$. Thus $h(A)<a-\frac{1}{2}$, contrary to assumption.

Now suppose $h(A) \leqslant-b-\frac{1}{2}$. Then $g(A) \leqslant-b+\frac{1}{2}$ and if $B \subset A \cap S$,

$$
-b+\frac{1}{2} \geqslant g(A) \geqslant g(B)+g(A \backslash B)-1 .
$$

Hence $g(B) \leqslant \frac{3}{2}$ and $\phi(A \cap S) \leqslant 2 \frac{1}{2}$. Thus, $\lambda(A \cap S)<\frac{1}{2}$ and hence

$$
h(A)>g(A)-\frac{1}{2}>-b-\frac{1}{2},
$$

contrary to assumption.

Now $V(h)<a+b$, contrary to assumption to $\mu$. We conclude that for every $\lambda$ : $2^{S} \rightarrow \mathbf{R}$ with $\lambda(S)=1$ then there exists $A \in \mathcal{C}$ with $\lambda(A) \geqslant \frac{1}{2}$. By Proposition 3.2 this implies $J(\mathcal{C}) \geqslant \frac{1}{2}$. 
Now we claim that since $\mathcal{C}$ is finite there exist $C_{1}, \ldots, C_{m} \in \mathcal{C}$ so that

$$
\frac{1}{2} 1_{\Omega} \leqslant \frac{1}{m} \sum_{i=1}^{m} 1_{C_{i}}
$$

In fact $J(\bigodot)^{-1}$ is the solution of the linear programming problem:

$$
\begin{gathered}
\text { Minimize: } \sum_{C \in e} x_{C} \\
\text { Constraints: } \sum_{C \in e_{C} x_{C} 1_{C}(\omega) \geqslant 1, \quad \omega \in \Omega,} x_{C} \geqslant 0 .
\end{gathered}
$$

Any basic feasible solution $\left(\bar{x}_{C}: C \in \mathcal{C}\right)$ of this problem has $\bar{x}_{C}$ rational for every $C \in \mathcal{C}$. (See, for example, [20, p. 14].) Thus $J(\mathcal{C})$ is rational and

$$
\sum_{C \in \bigodot} J(e) \bar{x}_{C} 1_{C} \geqslant \frac{1}{2} 1_{\Omega}
$$

Now by choosing $m$ as a greatest common denominator of $\left(J(\bigodot) \bar{x}_{C}: C \in \mathcal{C}\right)$ and repeating sets as required we can find $C_{1}, \ldots, C_{m} \in \mathcal{C}$ with

$$
\frac{1}{m} \sum_{i=1}^{m} 1_{C_{i}} \geqslant \frac{1}{2} 1_{\Omega}
$$

Now by Lemma 3.1 for any $r \in \mathbf{N}$,

$$
\frac{9}{2} \geqslant \frac{1}{m} \cdot \sum_{i=1}^{m} \phi\left(C_{i}\right) \geqslant(a+1)-2 r-(a-1) \theta\left(r, \frac{1}{2}\right) \text {. }
$$

If $r$ is chosen so that $\theta\left(r, \frac{1}{2}\right)<1$,

$$
a \leqslant\left(7+4 r-2 \theta\left(r, \frac{1}{2}\right)\right) / 2\left(1-\theta\left(r, \frac{1}{2}\right)\right) .
$$

Proposition 2.3(i) (depending only on Proposition 2.1) shows there exists $r \in \mathbf{N}$ so that $\theta\left(r, \frac{1}{2}\right)<1$. However 2.3(ii) gives a better estimate (depending on 2.2), i.e. $\theta\left(6, \frac{1}{2}\right) \leqslant \frac{2}{3}$. Hence

$$
a \leqslant \frac{7+24-4 / 3}{2 / 3}=\frac{89}{2}<45 .
$$

REMARKs. The problem of determining the best constant $K$ is unsolved. B. Pawlik has communicated to the authors an example to show that $K \geqslant \frac{3}{2}$ is necessary.

5. The control measure problem. Let $X$ be an $F$-space, and let $\mathcal{Q}$ be a $\sigma$-algebra of sets. Let $\mu: \mathbb{Q} \rightarrow X$ be any countably additive vector measure. A control measure $\lambda$ for $\mu$ is a countably additive scalar measure $\lambda: \mathcal{Q} \rightarrow \mathbf{R}$ such that $\lambda\left(A_{n}\right) \rightarrow 0$ implies $\left\|\mu\left(A_{n}\right)\right\| \rightarrow 0$. It is well known that the Maharam problem is equivalent to the problem of the existence of a control measure for a countably additive vector measure. In this section we show how the results of $\$ 3$ give us some positive results.

THEOREM 5.1. Suppose $\mu: Q \rightarrow X$ is a countably additive vector measure with relatively compact range. Then $\mu$ has a control measure. 
Proof. We define for $A \in \mathbb{Q}$,

$$
\|\mu\|(A)=\sup _{B \subset A}\|\mu(B)\| .
$$

Then $\|\mu\|$ is a submeasure on $Q$. We claim that $\|\mu\|$ is uniformly exhaustive.

Given $\varepsilon>0$ pick an integer $r$ so that $\left\|r^{-1} \mu(A)\right\| \leqslant \frac{1}{2} \varepsilon$ for $A \in \mathbb{Q}$; this is possible since $\mu(\mathbb{Q})$ is relatively compact and hence bounded. Pick $N \in \mathbf{N}$ so that $\mu(\mathbb{Q})$ can be covered by $N$ sets $K_{1}, \ldots, K_{n}$ of diameter at most $\varepsilon / 2 r$. Let $A_{1}, \ldots, A_{r N}$ be disjoint sets in $Q$, and suppose $\left\|\mu\left(A_{i}\right)\right\|>\varepsilon, i=1,2, \ldots, r N$. Then there exists $j \leqslant N$ and $i_{1}<i_{2}<\cdots<i_{r}$ so that $\mu\left(A_{i_{l}}\right) \in K_{j}$ for $l=1,2, \ldots, r$. Thus

$$
\left\|\mu\left(A_{i_{l}}\right)-\mu\left(A_{i_{1}}\right)\right\| \leqslant \varepsilon / 2 r
$$

and hence

$$
\left\|\mu\left(\bigcup_{l=1}^{r}\right) A_{i_{l}}-r \mu\left(A_{i_{1}}\right)\right\| \leqslant \varepsilon / 2 .
$$

Thus

$$
\left\|r^{-1} \mu\left(\bigcup_{l=1}^{r} A_{i_{l}}\right)-\mu\left(A_{i_{1}}\right)\right\| \leqslant \varepsilon / 2
$$

and so

$$
\left\|\mu\left(A_{i_{1}}\right)\right\| \leqslant \varepsilon
$$

contrary to assumption.

We conclude that

$$
\min _{1 \leqslant i \leqslant r N}\|\mu\|\left(B_{i}\right) \leqslant \varepsilon
$$

for any collection of disjoint sets $B_{1}, \ldots, B_{r N}$.

Now let $\lambda$ be a measure equivalent to $\|\mu\|$. Then if $A_{n} \downarrow \varnothing,\|\mu\|\left(A_{n}\right) \downarrow \varnothing$, i.e. $\lambda$ is countably additive, and clearly $\lambda$ controls $\mu$.

Corollary 5.2. If, in addition to the assumptions of the theorem, $\mu$ is nonatomic, then $\overline{\mu(\mathbb{Q})}$ is a compact convex set which is locally convex.

Proof. The fact that $\overline{\mu(\mathbb{Q})}$ is convex is shown in [9] for quasi-Banach spaces, but the argument goes through unchanged. Now since $\mu$ has a control measure, the set $\overline{c o} \mu(\mathbb{Q})$ is quasiconvex in the sense of [6, Theorem 4.1], i.e. it has enough continuous affine functionals to separate points. Hence being compact it is locally convex.

REMARKS. Corollary 5.2 has a geometrical interpretation. There are compact convex sets which are not locally convex $[16,17]$ and do not satisfy the Krein-Milman Theorem. However the closure of the range of a vector measure (sometimes called a zonoid) can never be such a set.

Now suppose $X$ is a quasi-Banach space. We shall suppose $X$ is equipped with a quasinorm $\|\cdot\|$ which is $p$-subadditive where $0<p<1$. We say that $l_{\infty}$ is (crudely) finitely representable in $X$ if there exists $c>0$ so that for every $n \in \mathbf{N}$ there is a linear embedding $T: l_{\infty}^{n} \rightarrow X$ so that $c\|x\| \leqslant\|T x\| \leqslant\|x\|, x \in l_{\infty}^{n}$. 
THEOREM 5.3. Let $X$ be a quasi-Banach space and suppose $l_{\infty}$ is not finitely representable in $X$. Then every countably additive vector measure $\mu: \mathbb{Q} \rightarrow x$ has a control measure.

Proof. Suppose $\mu: \mathbb{Q} \rightarrow X$ has no control measure. First we observe (see [19]) that $\operatorname{co} \mu(\mathbb{Q})$ is bounded so that if $c_{1}, \ldots, c_{n} \in \mathbf{R}$ and $A_{1}, \ldots, A_{n} \in \mathbb{Q}$ are disjoint,

$$
\left\|\sum_{i=1}^{n} c_{i} \mu\left(A_{i}\right)\right\| \leqslant M \max _{1 \leqslant i \leqslant n}\left|c_{i}\right| .
$$

If $\phi(A)=\sup _{B \subset A}\|\mu(B)\|^{p}$ then $\phi$ is a submeasure which cannot be uniformly exhaustive. Hence for some $\delta>0$ and any $n \in \mathbf{N}$ we can find disjoint $\left(A_{n, i}: 1 \leqslant i \leqslant\right.$ $n)$ in $Q$ so that $\left\|\mu\left(A_{n, i}\right)\right\| \geqslant \varepsilon$. Define $T_{n}: l_{\infty}^{n} \rightarrow X$ by

$$
T_{n}(x)=M^{-1} \sum_{i=1}^{n} x_{i} \mu\left(A_{n, i}\right) .
$$

Then $\left\|T_{n}\right\| \leqslant 1$ and $\left\|T_{n}\left(e_{k}\right)\right\| \geqslant M^{-1} \delta$ for each basis vector $e_{k}(1 \leqslant k \leqslant n)$. Now by a theorem of Drewnowski [3], $l_{\infty}$ is finitely representable in $X$, contrary to assumption.

6. Twisted sums. In [7] an $F$-space $X$ was defined to be a $K_{\text {-space }}$ if whenever $Y$ is an $F$-space with a one-dimensional subspace (a line) $L \subset Y$ so that $Y / L \cong X$, then $L$ is complemented in $Y$, so that $Y \cong X \oplus \mathbf{R}$. In [7] it is shown that the Banach space $l_{1}$ is not a $\mathcal{K}_{\text {-space }}$ (see also $[15,18]$ ) while $l_{p}(1<p<\infty)$ is a $K_{\text {-space. }}$ The problem raised in [7] is to determine whether $c_{0}$ is a $\mathcal{K}_{\text {-space; }}$ in this section we show that Theorem 4.1 shows that $c_{0}$ is a $\mathcal{K}_{\text {-space. }}$

More generally we recall that a Banach space $X$ is an $\varrho_{\infty}$-space if there is a constant $c \geqslant 1$ such that for every subspace $F$ of $X$ with $\operatorname{dim} F<\infty$ there is a further subspace of $G$ of $X$ with $G \supset F$ and $\operatorname{dim} G=m<\infty$ and a linear isomorphism $T: G \rightarrow l_{\infty}^{m}$ so that $\|T\| \cdot\left\|T^{-1}\right\| \leqslant c$.

If $X$ is a Banach space then a functional $f: X \rightarrow \mathbf{R}$ is called quasilinear if for some constant $\Delta=\Delta(f)$ and any $x_{1}, x_{2} \in X$,

(i) $\left|f\left(x_{1}+x_{2}\right)-f\left(x_{1}\right)-f\left(x_{2}\right)\right| \leqslant \Delta\left(\left\|x_{1}\right\|+\left\|x_{2}\right\|\right), x_{1}, x_{2} \in X$,

(ii) $f(t x)=t f(x), t \in \mathbf{R}, x \in X$.

We recall Proposition 3.3 of [7].

Proposition 6.1. Let $X$ be a Banach space. Then $X$ is a K -space if and only if for every quasilinear map $f: X \rightarrow \mathbf{R}$ there is a linear (not necessarily continuous) functional $h: X \rightarrow \mathbf{R}$ with $|f(x)-h(x)| \leqslant L\|x\|, x \in X$, for some constant $L$.

We observe that if $f$ is quasilinear and $x_{1}, \ldots, x_{n} \in X$ then

$$
\left|f\left(\sum_{i=1}^{n} x_{i}\right)-\sum_{i=1}^{n} f\left(x_{i}\right)\right| \leqslant \Delta\left(\sum_{k=1}^{n} k\left\|x_{k}\right\|\right) .
$$

This is proved by a simple induction (cf. Lemma 3.2 of [7]).

Let $\Omega$ be a finite set and let $l_{\infty}(\Omega)$ denote the space of all real functions on $\Omega$ with the usual sup-norm. 
Proposition 6.2. Let $f: l_{\infty}(\Omega) \rightarrow \mathbf{R}$ be a quasilinear map. Then there is a linear functional $h: l_{\infty}(\Omega) \rightarrow \mathbf{R}$ with $|f(x)-h(x)| \leqslant 100 \Delta\|x\|$ where $\Delta=\Delta(f)$.

Proof. The set function $A \mapsto f\left(1_{A}\right)$ for $A \subset \Omega$ is $\Delta$-approximately additive and so there is an additive set-function $\mu$ with $|f(A)-\mu(A)| \leqslant 45 \Delta, A \in 2^{\Omega}$ (Theorem 4.1). Let $h$ be the natural linear extension of $\mu$ to $l_{\infty}(\Omega)$. Then $g=f-h$ is quasilinear and $\left|g\left(1_{A}\right)\right| \leqslant 45 \Delta, A \in 2^{\Omega}$.

If $x \in l_{\infty}(\Omega)$ then $x=\Sigma_{\omega \in \Omega} x(\omega) e_{\omega}$ where $e_{\omega}=1_{\{\omega\}}$. Thus

$$
\left|g(x)-\sum_{\omega \in \Omega} x(\omega) g\left(e_{\omega}\right)\right| \leqslant|\Omega|^{2} \Delta\|x\|
$$

and so

$$
|g(x)| \leqslant\left(|\Omega|^{2}+45|\Omega|\right) \Delta\|x\| .
$$

Now suppose $0 \leqslant x \leqslant 1_{\Omega}$. Then for suitable $A_{1}, \ldots, A_{m} \in 2^{\Omega}$,

$$
\left\|x-\sum_{k=1}^{m} \frac{1}{2^{k}} 1_{A_{k}}\right\| \leqslant 2^{-m}
$$

and

$$
\left|g\left(\sum_{k=1}^{m} \frac{1}{2^{k}} 1_{A_{k}}\right)-\sum_{k=1}^{m} \frac{1}{2^{k}} g\left(1_{A_{k}}\right)\right| \leqslant \Delta \sum_{k=1}^{m} \frac{k}{2^{k}} \leqslant 2 \Delta .
$$

Thus

$$
\left|g\left(\sum_{k=1}^{m} \frac{1}{2^{k}} 1_{A_{k}}\right)\right| \leqslant 47 \Delta .
$$

Hence

$$
|g(x)| \leqslant 47 \Delta+2^{-m}\left(|\Omega|^{2}+45|\Omega|\right) \Delta+\Delta\left(1+2^{-m}\right) .
$$

Letting $m \rightarrow \infty,|g(x)| \leqslant 48 \Delta$.

Now for any $x \in l_{\infty}(\Omega)$ with $x=u-v$, where $u, v \geqslant 0$, and $\|u\|,\|v\| \leqslant\|x\|$, then

$$
\begin{aligned}
|g(x)| & \leqslant|g(u)|+|g(v)|+\Delta(\|u\|+\|v\|) \\
& \leqslant(48 \Delta+48 \Delta+2 \Delta)\|x\|<100 \Delta\|x\|
\end{aligned}
$$

as required.

THEOREM 6.3. Let $X$ be a Banach space with is an $\mathcal{L}_{\infty}$-space. Then $X$ is a F-space.

Proof. Suppose that $f: X \rightarrow \mathbf{R}$ is a quasilinear map with $\Delta=\Delta(f)$. There is a constant $c \geqslant 1$ so that whenever $F \subset X$ is a finite-dimensional subspace, there is a finite-dimensional subspace $G$ with $G \supset F$ and a linear isomorphism $T: G \rightarrow l_{\infty}^{m}$ with $\left\|T^{-1}\right\| \leqslant c$.

Let $F$ be any finite-dimensional subspace of $X$ and choose $G \supset F$ as above. Then $f \circ T^{-1}$ is quasilinear on $l_{\infty}^{m}$ with $\Delta\left(f \circ T^{-1}\right) \leqslant\left\|T^{-1}\right\| \Delta$.

Hence there is a linear map $h: l_{\infty}^{m} \rightarrow \mathbf{R}$ with

$$
\left\|f\left(T^{-1} u\right)-h(u) \mid \leqslant 100\right\| T^{-1}\|\Delta\| u \|, \quad u \in l_{\infty}^{m} .
$$


Define $d_{F}: F \rightarrow \mathbf{R}$ by $d_{F}(x)=h(T x)$. Then

$$
\left|f(x)-d_{F}(x)\right| \leqslant 100\left\|T^{-1}\right\| \Delta\|T x\| \leqslant 100 c \Delta\|x\|, \quad x \in F .
$$

Ordering the finite-dimensional subspaces of $X$ by inclusion we obtain a net of functions $d_{F}^{*}: X \rightarrow \mathbf{R}$ where $d_{F}^{*}(x)=d_{F}(x)(x \in F)$ and $d_{F}^{*}(x)=0$ for $x \notin F$. For any $x \in X$,

$$
\left|d_{F}^{*}(x)\right| \leqslant 100 c \Delta\|x\|+|f(x)|
$$

and $d_{F}^{*}$ has a subnet converging pointwise to a linear functional $d: X \rightarrow \mathbf{R}$. Clearly

$$
|f(x)-d(x)| \leqslant 100 c \Delta\|x\|, \quad x \in X .
$$

COROllary 6.4. The Banach space $c_{0}$ is a K-space.

Theorem 6.5. Let $X$ be a quotient of $\mathcal{L}_{\infty}$-space. Then $X$ is a K $\mathcal{K}$-space.

Proof. Let $Y$ be an $\mathcal{L}_{\infty}$-space, $Q: Y \rightarrow X$ a quotient mapping, and $f: X \rightarrow \mathbf{R}$ a quasilinear map. Then $f \circ Q$ is quasilinear on $Y$ so that there exists a linear map $h$ : $Y \rightarrow \mathbf{R}$ with $|f \circ Q(y)-h(y)| \leqslant L\|y\|, y \in Y$, for some constant $L<\infty$. If $y \in$ $Q^{-1}(0),|h(y)| \leqslant L\|y\|$, and so by the Hahn-Banach theorem there is a continuous linear functional $y^{*} \in Y^{*}$ with $\left\|y^{*}\right\| \leqslant L$ so that $y^{*}(y)=h(y)$ for $y \in Q^{-1}(0)$.

Now $h-y^{*}=d \circ Q$ for some linear functional $d$ on $X$.

If $x \in X$ and $x=Q y$ then

$$
|f(x)-d(x)|=|f(Q y)-d(Q y)| \leqslant|f(Q y)-h(y)|+\left|y^{*}(y)\right| \leqslant 2 L\|y\| .
$$

Hence

$$
|f(x)-d(x)| \leqslant 2 L\|x\|, \quad x \in X
$$

and $X$ is a $\mathcal{K}$-space.

REMARK. We recall ([7, Theorem 4.10]) that if $X$ is a $\mathcal{K}$-space and $Y$ is any $F$-space with a locally convex subspace $N$ such that $Y / N \cong X$, then $Y$ is itself locally convex.

We conjecture that a Banach space $X$ is a $\mathcal{K}_{-}$-space if and only if $l_{\infty}$ is not finite representable in $X^{*}$ (or equivalently $X$ does not contain uniformly complemented $l_{1}^{n}$ 's). In this context we remark that the assumptions of Theorem 4.7 of [7] already imply this conclusion.

\section{REFERENCES}

1. J. P. R. Christensen, Some results with relation to the control measure problem, Vector Space Measures and Applications. II, Lecture Notes in Math., vol. 645, Springer-Verlag, Berlin and New York, 1978, pp. 27-34.

2. J. P. R. Christensen and W. Herer, On the existence of pathological submeasures and the construction of exotic topological groups, Math. Ann. 213 (1975), 203-210.

3. L. Drewnowski, Un théorème sur les opérateurs de $l_{\infty}(\Gamma)$, C. R. Acad. Sci. Paris Sér. A 281 (1976), 967-969.

4. O. Gabber and Z. Galil, Explicit constructions of linear size superconcentrators, J. Comput. System Sci. 22 (3) (1981), 407-420.

5. P. Hall, On representatives of subsets, J. London Math. Soc. 10 (1935), 26-30.

6. N. J. Kalton, Linear operators whose domain is locally convex, Proc. Edinburgh Math. Soc. 20 (1976), 293-299.

7.

8. Univ., 1980, G. A. Goldin and R. F. Wheeler, editors), DeKalb, Illinois, 1981. 
9. , Isomorphisms between spaces of vector-valued continuous functions, Proc. Edinburgh Math. Soc. (to appear).

10. J. L. Kelley, Measures on Boolean algebras, Pacific J. Math. 9 (1959), 1165-1177.

11. D. Maharam, An algebraic characterization of measure algebras, Ann. of Math. (2) 48 (1947), 154-167.

12. M. S. Pinsker, On the complexity of a concentrator, 318/1-318/4, 7th Internat. Telegraphic Conf., Stockholm, June 1973.

13. N. Pippenger, Superconcentrators, SIAM J. Comput. 6 (1977), 298-304.

14. V. A. Popov, Additive and subadditive functions on Boolean algebras, Siberian Math. J. 17 (1976), 331-339. (Russian)

15. M. Ribe, Examples for the non-locally convex three-space problem, Proc. Amer. Math. Soc. 73 (1979), 351-355.

16. J. W. Roberts, Pathological compact convex sets in the spaces $L_{p}, 0<p<1$, The Altgeld Book, University of Illinois, 1976.

17. __ A compact convex set with no extreme points, Studia Math. 60 (1977), 255-266.

18. A non-locally convex $F$-space with the Hahn-Banach approximation property, Banach Spaces of Analytic Functions, Lecture Notes in Math., vol. 604, Springer-Verlag, Berlin and New York, 1977, pp. 76-82.

19. S. Rolewicz and C. Ryll-Nardzewski, On unconditional convergence in linear metric spaces, Colloq. Math. 17 (1967), 327-331.

20. M. Simonard, Linear programming, Prentice-Hall, Englewood Cliffs, N. J., 1967.

21. M. Talagrand, A simple example of a pathological submeasure, Math. Ann. 252 (1980), 97-102.

Department of Mathematics, University of Missouri, Columbia, Missouri 65211

Department of Mathematics, University of South Carolina, Columbia, South Carolina 29208 